\title{
Use of digital retinal camera to detect prevalence and severity of diabetic retinopathy in a screening program for type 2 diabetic refugees in Palestine
}

\author{
Riyad G. Banayot $\odot$
}

St John Eye Hospital, Hebron, Palestine

\begin{abstract}
BACKGROUND: The purpose of this study was to determine the prevalence of diabetic retinopathy among Palestinian refugees serviced by the Diabetic Retinopathy Screening Program in the Occupied Palestinian Territories (DRS-OPT).

MATERIAL AND METHODS: This is a retrospective study of retinal images of 1891 diabetic patients in 15 urban UNRWA clinics participating in the DRS-OPT program in Palestine over 12 months. A nonmydriatic Canon CR-2 fundus retinal camera was used to capture two 450 non-stereo fundus images for each eye. Qualified graders (nurses) performed the grading based on the DRS-OPT grading system.

RESULTS: Out of the 1891 diabetic patients screened, 1694 had at least one gradable eye. $16 \%$ of patients had diabetic retinopathy $(5.7 \%$ had mild nonproliferative diabetic retinopathy, $4.3 \%$ had moderate nonproliferative diabetic retinopathy, $1.1 \%$ had severe, moderate nonproliferative diabetic retinopathy, and $1.7 \%$ had proliferative diabetic retinopathy. Maculopathy without retinopathy amounted to 3\%. Other findings included the identification of blinding diseases such as age-related macular degeneration and optic disc glaucomatous cupping.

CONCLUSIONS: The retinopathy screening program using a nonmydriatic fundus camera identified diabetic retinopathy in $16 \%$ of diabetic Palestinian refugees. A total of $72 \%$ of these patients were diabetics with nonproliferative retinopathy. This program can be used to prevent progression by facilitating the education of patients and early intervention.
\end{abstract}

KEY-WORDS: diabetic retinopathy; screening; digital camera; prevalence; Palestine

Ophthalmol J 2021; Vol. 6, 107-112

\section{INTRODUCTION}

The United Nations Relief and Works Agency for Palestine refugees in the Near East (UNRWA) stated in their 2019 health department annual report that the prevalence of diabetes mellitus in the West Bank was $17.1 \%$ among served popula- tion $\geq 40$ years and that 6,264 diabetes mellitus type 2 patients were registered [1]. In 2020, the International Diabetes Federation reported a 6.7\% prevalence rate of diabetes in adults in Palestine [2].

The prevalence of diabetic retinopathy (DR) has been previously reported in several populations. Data 
pooled from 35 studies (1980-2008) estimated the overall prevalence as $34.6 \%$ for any diabetic retinopathy (mild or moderate nonproliferative diabetic retinopathy NPDR), $6.96 \%$ for proliferative diabetic retinopathy (PDR), $6.81 \%$ for diabetic macular edema/maculopathy (DME), and $10.2 \%$ for vision-threatening diabetic retinopathy [3].

In 2012, a collaborative project, called Diabetic Retinopathy Screening Program in the Occupied Palestinian Territories (DRS-OPT), was set up between St. John of Jerusalem Eye Hospital and UNRWA for a 3-year screening, treatment, and management for diabetic retinopathy among diabetic patients in East Jerusalem and the West Bank including the refugee population of the Southern districts of the West Bank (Bethlehem and Hebron) [4].

Following the initial three years of DRS-OPT, UNRWA assumed ownership of the program and continued the annual screening of their patients at their clinics. The aim was to identify any diabetic retinopathy and facilitate referral to specialized eye services.

Many countries have adopted national diabetic retinopathy screening programs. Studies have shown that screening programs using digital cameras with or without pupil dilation may assist in the early detection of diabetic retinopathy. Diabetic retinopathy screening aims to reduce the risk of vision impairment and blindness among asymptomatic people with diabetes through the prompt identification and effective treatment of sight-threatening diabetic retinopathy [5].

The objective of our study is to determine the prevalence of diabetic retinopathy among Palestinian refugees serviced by the Diabetic Retinopathy Screening Program in the Occupied Palestinian Territories.

\section{MATERIAL AND METHODS}

This study is a retrospective case series of retinal images of patients with diabetes in 15 urban UNRWA clinics in Palestine. UNRWA's diabetic registry was used to identify diabetic patients. All patients 18 years or older diagnosed with type 2 diabetes mellitus were made aware of the screening program. The clinic personnel contacted patients in their respective clinics and by word-of-mouth in the camps.

A total of 1891 patients attended the clinic-based mobile screening units over 12 months. UNRWA
Ethics Committee provided approval and permission to conduct the study. No informed consent was obtained because the names of patients were masked in data forms to maintain confidentiality. Participants who had diabetes mellitus type 2 and at least one gradable eye were included in the study. Exclusion criteria included patients with missing images or when both images were ungradable.

The screening unit personnel consisted of two trained nurses. Personnel at the screening unit used a structured form to collect demographic and clinical information. All screening centers used this form. Nurses surveyed patients for the duration of diabetes, current treatments, including medications for other systemic diseases. Snellen charts were used to measure visual acuity. Each patient underwent color fundus photography using the nonmydriatic Canon CR-2 retinal camera captured by trained nurses. Each eye was photographed using two 450 non-stereo fundus fields.

Following image capturing, trained nurses graded the images on-site at the time of screening. Training of graders was performed according to the DRS-OPT program. Good/adequate quality images (gradable) showed: one fovea centered field (center of fovea $\leq 1$ disc diameter (DD) from the center of the image, center of fovea $>2 \mathrm{DD}$ from the edge of the image, vessels are visible within 1DD of the center of fovea and vessels were visible across $>90 \%$ of the image), the second disc centered field (center of disc $\leq 1 \mathrm{DD}$ from the center of the image, complete optic disc $>2 \mathrm{DD}$ from the edge of the image, fine vessels were visible on the surface of disc and vessels visible across $>90 \%$ of the image). Images that did not meet the quality standards were considered ungradable. The following DR characteristics of the retinal fundus images were evaluated carefully: microaneurysms, dot-blot hemorrhages, hard exudates, venous beading, intraretinal microvascular abnormalities, central macular edema, disc neovascularization, neovascularization elsewhere, or preretinal hemorrhage. A minimum of $60 \%$ of the macula should be visible for images to be included in the study. If it was difficult to assess the presence or absence of DR due to inadequate image quality, the image was excluded from the study.

The eye with the more advanced level of DR defined the severity level of DR for each patient. The grading level of the patient's retinopathy was determined by the stage of DR in the gradable eye if the other eye was ungradable. Any other ocular pathology, such as hypertensive retinopathy, glaucomatous 
disc cupping, and age-related macular degeneration (AMD), was also noted.

The DRS-OPT grading system was used to grade images:

No retinopathy/R0 (no pathological lesions in the retina).

Mild nonproliferative retinopathy/R1 (microaneurysms or dot-blot hemorrhages).

Moderate nonproliferative retinopathy/R2 (one lesion of hard exudates, cotton wool spots, or flame-shaped hemorrhages).

Severe nonproliferative retinopathy/R2 (microaneurysms $>20$ in each quadrant or venous beading in two quadrants or intra-retinal microvascular abnormalities).

Proliferative retinopathy/R3 (new vessels on disc or new vessels elsewhere or preretinal hemorrhage or vitreous hemorrhage or fibrous proliferation).

No maculopathy/M0 (no pathological lesions of the macula).

Maculopathy (diabetic macular edema)/M1 (exudate $<$ or $=1$ disc diameter of the center of the fovea or circinate or group of exudates within macula or microaneurysm or hemorrhage $<$ or $=1$ disc diameter of the center of the fovea or retinal thickening $<$ or $=1$ disc diameter of the center of the fovea).

Definition of sight-threatening retinopathy includes the presence of diabetic retinopathy (R2) or worse, or the presence of maculopathy (M1). Any retinopathy (R1, R2, R3, and M1) or other findings, e.g., vitreous hemorrhage and end-stage disease) detected by the primary grader were sent for secondary grading performed by another grader. Images were submitted to an ophthalmologist for arbitration when disagreement occurred between the primary and secondary grader. An earlier study conducted by the author [4] found the interobserver agreement was $80 \%$ or better for the overall outcome.

The primary objective of the screening program is to detect the maximum number of cases of sight-threatening retinopathy and refer them for further examination and management by an ophthalmologist while retaining those with a non-sight-threatening disease under periodic review. DR presence and grade were used for referral recommendations. For patients without DR changes or mild NPDR, follow-up screening was scheduled within 12 months. Referrals to an ophthalmologist were made for patients with moderate NPDR or maculopathy. Patients with severe NPDR or PDR were urgently referred to an ophthalmologist. Referrals to an ophthalmologist for a dilated fundus examination were made for patients with ungradable images due to poor quality or for other lesions. The Palestinian Ophthalmic Society devised a protocol (adapted from two international ophthalmological organizations, the American Academy of Ophthalmology [6], International Council of Ophthalmology [7]) to care for diabetic retinopathy patients.

\section{RESULTS}

A total of 1891 diabetic patients attended the screening units during a 12-month period. Of these, in 197 patients, images were ungradable, and patients were excluded from the study. Consequently, the study included 1694 patients. Of the patients evaluated, $84 \%$ had no retinopathy in either eye (Tab. 1), which reveals a prevalence rate of $16 \%$ for DR. Of the 271 patients who had retin-

Table 1. Diabetic retinopathy severity

\begin{tabular}{|c|c|c|c|}
\hline \multicolumn{2}{|l|}{ Diagnosis by patient $(n=1694)$} & No. & $\%$ \\
\hline No retinopathy & No retinopathy, no maculopathy & 1423 & 84 \\
\hline \multirow{7}{*}{ Retinopathy (non proliferative or proliferative) } & Mild NPDR, no maculopathy & 46 & 2.7 \\
\hline & Mild NPDR, maculopathy & 52 & 3.1 \\
\hline & Moderate NPDR, no maculopathy & 22 & 1.3 \\
\hline & Moderate NPDR, maculopathy & 52 & 3.1 \\
\hline & Severe NPDR, no maculopathy & 2 & 0.1 \\
\hline & Severe NPDR, maculopathy & 21 & 1.2 \\
\hline & PDR, maculopathy & 29 & 1.7 \\
\hline Maculopathy & Maculopathy without retinopathy & 47 & 2.8 \\
\hline
\end{tabular}

NPDR — non proliferative diabetic retinopathy; PDR — proliferative diabetic retinopathy 


\begin{tabular}{|l|c|c|}
\hline Table 2. Other ocular findings \\
\hline Other lesions (1694 patients) & No. & $\%$ \\
\hline Previous laser marks & 53 & 3.1 \\
\hline Age-related macular degeneration & 21 & 1.2 \\
\hline Myopic degeneration & 20 & 1.2 \\
\hline Optic disc glaucomatous cupping & 9 & 0.5 \\
\hline Congenital night blindness & 9 & 0.5 \\
\hline Toxoplasmosis scar & 5 & 0.3 \\
\hline Other & 7 & 0.4 \\
\hline Total & 124 & 7.3 \\
\hline
\end{tabular}

opathy or maculopathy changes, $2.7 \%$ had mild NPDR without maculopathy, and $3.1 \%$ had mild NPDR with maculopathy in the more severe eye. Sight-threatening retinopathy (moderate NPDR, severe NPDR, PDR, and maculopathy) represented $13.3 \%$ cases. Of 271 patients who had retinopathy: $72 \%$ had NPDR, $10.7 \%$ had PDR, and $17.3 \%$ had maculopathy (Tab. 2).

In addition to diabetic retinopathy, $7.3 \%$ of patients had other potentially vision-threatening findings during image evaluation (Tab. 2). These pathologies included previous laser marks (53 patients), age-related macular degeneration $(21 \mathrm{pa}$ tients), myopic degeneration (20 patients), optic disc glaucomatous cupping (9 patients), congenital night blindness (9 patients), toxoplasmosis scars (5 patients), other findings (hypertensive retinopathy, optic disc atrophy, macular hole, asteroid hyalosis, drusen, myelinated nerve fiber layer).
Referral of patients followed the DRS-OPT program recommendations: annual re-screening was scheduled for $77.7 \%$ of patients, referral of $26.1 \%$ of patients for an ophthalmologist for a dilated fundus assessment for retinopathy, or other lesions or ungradable images, $2.7 \%$ of patients were urgently referred for management by an ophthalmologist for their retinopathy (Tab. 3).

\section{DISCUSSION}

To our knowledge, no previous study has been published regarding DR prevalence in type $2 \mathrm{DM}$ patients in Palestine. Previous studies in the surrounding countries range from $15.8 \%$ [8] to $42 \%$ [9]. Table 4 summarizes similar epidemiologic studies on DR prevalence. Our data revealed a very close prevalence rate of DR to that reported from Israel (15.8\%) [9] but lower than rates in Jordan (34.1\%) [10], Lebanon (35\%) [11], or Egypt (42\%) [9]. Our results are close to the CURES study (17.6\%) [12] and Aus-Diab study group (15.3\%) [13], although both studies were population-based and not clinic-based like ours. The percentage of patients with PDR was $1.7 \%$, constituting about $10.7 \%$ of all patients with DR (Tab. 1). This distribution is higher than that reported from Israel but lower than that from Jordan and Lebanon. DME/maculopathy (alone) reached a prevalence of 3\%, a figure higher than that recorded in Israel but lower than that of Lebanon. Of note, maculopathy with retinopathy reached a prevalence of $9.1 \%$ (Tab. 1).

Table 3. Referral recommendations

\begin{tabular}{|l|l|c|c|}
\hline Diagnosis (1891 patients) & Referral & No. & $\%$ \\
\hline No retinopathy, no maculopathy & Annual screening & 1423 & 75.3 \\
\hline Mild NPDR, no maculopathy & Annual screening & 46 & 2.4 \\
\hline Mild NPDR, maculopathy & Refer & 52 & 2.7 \\
\hline Moderate NPDR, no maculopathy & Refer & 22 & 1.2 \\
\hline Moderate NPDR, maculopathy & Refer & 52 & 2.7 \\
\hline Maculopathy without retinopathy & Refer & 47 & 2.5 \\
\hline Ungradable images & Refer & 197 & 10.4 \\
\hline Other lesions & Refer & 124 & 6.6 \\
\hline Severe NPDR, no maculopathy & Urgent refer & 2 & 0.1 \\
\hline Severe NPDR, maculopathy & Urgent refer & 21 & 1.1 \\
\hline PDR, maculopathy & Urgent Refer & 29 & 1.5 \\
\hline
\end{tabular}

NPDR - non proliferative diabetic retinopathy; PDR — proliferative diabetic retinopathy 
Table 4. Studies investigating diabetic retinopathy prevalence

\begin{tabular}{|l|c|c|c|c|}
\hline Author & Population & Prevalence of DR (\%) & Prevalence of DME (\%) & $\begin{array}{c}\text { DR patients with PDR } \\
\text { (\%) }\end{array}$ \\
\hline Herman et al. [8] & Egypt & 42 & N/A & N/A \\
\hline Salti et al. [9] & Lebanon & 35 & 8.4 & 26 \\
\hline Al-Amer et al. [6] & Jordan & 34.1 & N/A & 9.6 \\
\hline Banayot & Palestine & 16 & $3^{\#}$ & 1.7 \\
\hline Levy et al. [7] & Israel & 15.8 & 2.4 & 1.2 \\
\hline
\end{tabular}

*Current study. DR — diabetic retinopathy; DME — diabetic macular edema; PDR — proliferative diabetic retinopathy; N/A — not applicable; \#Prevalence of DME without retinopathy

An interesting finding in this study is that $72 \%$ of patients (195/271) who had diabetic retinopathy (Tab. 1) had nonproliferative diabetic retinopathy, which represents an opportunity for patient education and intervention to prevent further progression of retinopathy.

Identifying blinding diseases such as age-related macular degeneration and optic disc glaucomatous cupping through imaging and screening is a benefit to the screening program.

Screening programs offer the following advantages:

1. Service can be delivered in easily accessible locations (portable).

2. A trained technician can conduct the screening.

3. During the same visit, patients can optionally have an eye examination (slit-lamp is available).

4. The capacity of the screen can easily be increased.

5. An independent audit of images ensures high -quality screening and allows for evaluating screeners' performance.

6. Delivering, storing, grading, and communicating information to patients and health professionals is made more efficient by software/hardware.

7. The objective comparison of the same person, or between different people, examined at other times or by different professionals.

8. Suitable for patient education.

DR screening programs are very cost-effective from a socioeconomic perspective. The costs to society of ignoring prevention and the consequences, including supporting the blind, are between 7 and 20 times greater than the cost of prevention [14].

The study had several limitations, including that certain demographic and clinical data, such as age, gender, diabetes duration, history of other systemic diseases, medications, and glycemic control history, were not included in the analysis. As a result, we could not assess the effect of these factors on screen- ing results. Also, the study's population of Palestinian refugees in the West Bank may have added selection bias. The assessment of follow-up eye examinations and the efficacy of screening programs requires further research. The use of non-mydriatic fundus photographs is another study limitation. Nevertheless, in a study by Stellingwerf et al. [15], the sensitivity of two-field photography in identifying diabetic individuals with sight-threatening diabetic retinopathy was 95\% (specificity 99\%), and the sensitivity for detecting any diabetic retinopathy was $83 \%$ (specificity $88 \%$ ).

\section{Acknowledgments \\ NIL.}

\section{Competing interests}

NIL.

\section{REFERENCES}

9. United Nations Relief and Works Agency. Health Department Annual Report 2019. https://www.unrwa.org/2019UNRWAHealthAnnualR eport..

10. International Diabetes Federation. https://www.idf.org/our-network/ regions-members/middle-east-and-north-africa/members/44-palestine.html (June 1, 2021).

11. Yau JWY, Rogers SL, Kawasaki R, et al. Meta-Analysis for Eye Disease (META-EYE) Study Group. Global prevalence and major risk factors of diabetic retinopathy. Diabetes Care. 2012; 35(3): 556-564, doi: 10.2337/dc11-1909, indexed in Pubmed: 22301125.

12. Banayot R. Inter-grader agreement in the diabetic retinopathy screening program in palestine. Ibnosina J Med Biomed Sci. 2018; 10(6): 193, doi: 10.4103/ijmbs.ijmbs 6718.

13. World Health Organization 2020. Diabetic retinopathy screening: a short guide. https://apps.who.int/iris/bitstream/handle/10665/336660/ 9789289055321-eng.pdf.

14. Flaxel CJ, Adelman RA, Bailey ST, et al. Diabetic Retinopathy Preferred Practice Pattern ${ }^{\circledR}$. Ophthalmology. 2020; 127(1): P66-P6P145, doi: 10.1016/j.ophtha.2019.09.025, indexed in Pubmed: 31757498.

15. ICO Guidelines for Diabetic Eye Care. http://www.icoph.org/downloads/ICOGuidelinesforDiabeticEyeCare.pdf (June 1, 2021).

16. Levy J, Lifshitz T, Goldfarb D, et al. Screening for diabetic retinopathy with a mobile non-mydriatic digital fundus camera in southern Israel. Isr Med Assoc J. 2011; 13(3): 137-140, indexed in Pubmed: 21608332.

17. Herman WH, Aubert RE, Engelau MM, et al. Diabetes mellitus in Egypt: glycemic control and microvascular and neuropathic complications. Diabet Med. 1998; 15: 1045-1051, doi: 10.1002/(SICI)10969136(1998120)15:12<1045::AID-DIA696>3.0.CO;2-L, indexed in Pubmed: 9868980. 
18. Al-Amer RM, Khader Y, Malas S, et al. Prevalence and risk factors of diabetic retinopathy among Jordanian patients with type 2 diabetes. Digit J Ophthalmol. 2008; 14: 42-49, doi: 10.5693/djo.01.2008.013, indexed in Pubmed: 29440980.

19. Salti HI, Nasrallah MP, Taleb NM, et al. Prevalence and determinants of retinopathy in a cohort of Lebanese type II diabetic patients. Can J Ophthalmol. 2009; 44(3): 308-313, doi: 10.3129/i09-029, indexed in Pubmed: 19491987.

20. Rema M, Premkumar $S$, Anitha $B$, et al. Prevalence of diabetic retinopathy in urban India: the Chennai Urban Rural Epidemiology Study (CURES) eye study, I. Invest Ophthalmol Vis Sci. 2005; 46(7): 2328-2333, doi: 10.1167/iovs.05-0019, indexed in Pubmed: 15980218.
21. Tapp RJ, Shaw JE, Harper CA, et al. The Prevalence of and Factors Associated With Diabetic Retinopathy in the Australian Population. Diabetes Care. 2003; 26(6): 1731-1737, doi: 10.2337/diacare.26.6.1731, indexed in Pubmed: 12766102.

22. Stefánsson $E$, Bek T, Porta $M$, et al. Screening and prevention of diabetic blindness. Acta Ophthalmol Scand. 2000; 78(4): 374-385, doi: 10.1034/j.1600-0420.2000.078004374.x, indexed in Pubmed: 10990036.

23. Stellingwerf $\mathrm{C}$, Hardus PL, Hooymans JM. Two-field photography can identify patients with vision-threatening diabetic retinopathy: a screening approach in the primary care setting. Diabetes Care. 2001; 24(12): 2086-2090, doi: 10.2337/diacare.24.12.2086, indexed in Pubmed: 11723088. 